\title{
Common fixed point theorems on quasi-cone metric space over a divisible Banach algebra
}

\author{
Andreea Fulga ${ }^{1}$, Hojjat Afshari2 ${ }^{2 *}$ and Hadi Shojaat ${ }^{3}$
}

\section{"Correspondence:}

hojat.afshari@yahoo.com

${ }^{2}$ Department of Mathematics, Basic

Science Faculty, University of Bonab,

Bonab, Iran

Full list of author information is

available at the end of the article

\section{Springer}

\begin{abstract} point for the self-mappings defined on quasi-cone metric space over a divisible Banach algebra via an auxiliary mapping $\phi$.

Keywords: Common fixed point; $\psi$-operator; Quasi-cone metric; Quasi-Banach-valued metric space
\end{abstract}

In this manuscript, we investigate the existence and uniqueness of a common fixed

\section{Introduction and preliminaries}

The notion of metric has been extended in several ways by changing the axioms of the metric notion: quasi-metric, symmetric, dislocated metric, b-metric, 2-metric, D-metric, Smetric, G-metric, partial metric, ultra-metric, etc. We shall focus on cone metric space or more precisely, Banach-valued metric space. The idea of Banach-valued metric space was considered by several authors in distinct periods of the last century. This notion became popular and raised interest among researchers after the paper of Huang and X. Zhang [1] in 2007. Since then, a number of authors got the characterization of several known fixed point theorems in the context of Banach-valued metric space, such as, [2-20].

In this paper, we consider common fixed point theorems in the framework of the refined cone metric space, namely, quasi-cone metric space.

In what follows, we shall recall the basic notions and notations as well as the fundamental results.

Definition 1.1 ([21]) Suppose $\mathcal{E}$ is a real Banach algebra, that is, for $v, \omega, \eta \in \mathcal{E}, a \in \mathrm{R}$,

(a) $v(\omega z)=(v \omega) z$;

(b) $v(\omega+z)=v \omega+v z,(v+\omega) z=v z+\omega z$;

(c) $a(v \omega)=(a v) \omega=(a \omega) v$;

(d) $\|v \omega\| \leq\|v\|\|\omega\|$.

If Banach algebra $\mathcal{E}$ with unit element e, i.e. multiplicative identity e, is with $v \mathrm{e}=\mathrm{e} v=v$ for $v \in \mathcal{E}$, then $\|\mathrm{e}\|=1$.

(c) The Author(s) 2021. This article is licensed under a Creative Commons Attribution 4.0 International License, which permits use, sharing, adaptation, distribution and reproduction in any medium or format, as long as you give appropriate credit to the original author(s) and the source, provide a link to the Creative Commons licence, and indicate if changes were made. The images or other third party material in this article are included in the article's Creative Commons licence, unless indicated otherwise in a credit line to the material. If material is not included in the article's Creative Commons licence and your intended use is not permitted by statutory regulation or exceeds the permitted use, you will need to obtain permission directly from the copyright holder. To view a copy of this licence, visit http://creativecommons.org/licenses/by/4.0/. 
An element $v \in \mathcal{E}$ is said to be invertible if there exists $v^{-1} \in \mathcal{E}$ such that $v v^{-1}=v^{-1} v=\mathrm{e}$. Moreover, if every non-zero element of $\mathcal{E}$ has an inverse in $\mathcal{E}$, then $\mathcal{E}$ is called a divisible Banach algebra.

Proposition $1.2([22])$ Let $\mathcal{E}$ be a Banach algebra, $v$ an element in $\mathcal{E}$ and $\rho(v)$ the spectral radius of $v$. If $\rho(v)<1$ then $(\mathrm{e}-v)$ is invertible in $\mathcal{E}$ and

$$
(\mathrm{e}-v)^{-1}=\sum_{p=0}^{\infty} v^{p} .
$$

Remark $1.3([2]) \rho(v) \leq\|v\|$ for all $v$ in a Banach algebra $\mathcal{E}$.

Let $(\mathcal{E},\|\cdot\|)$ be a real algebra and $\mathrm{P}$ a closed subset of $\mathcal{E}$.

The set $\mathrm{P}$ is a cone if the following conditions hold:

$\left(c_{1}\right) \mathrm{P}$ is non-empty and $\mathrm{P} \neq\{\theta\}$;

$\left(c_{2}\right) \mathrm{a}_{1} v+\mathrm{a}_{2} \omega \in \mathrm{P}$ for all $v, \omega \in \mathrm{P}$ and $\mathrm{a}_{1}, \mathrm{a}_{2} \in(0, \infty)$;

$\left(c_{3}\right) \mathrm{P} \cap(-\mathrm{P})=\{\theta\}$.

Moreover, for a given cone $\mathrm{P} \subseteq \mathcal{E}$ we can consider a partial ordering $\leq$ such that $v \leq \omega$ if and only if $\omega-v \in P$. We write $v \ll \omega$ for $\omega-v \in$ intP and $v<\omega$ indicates that $v \leq \omega$ and $v \neq \omega$. The cone $P$ is called normal if there exists a constant $N>0$ such that $0 \leq v \leq \omega$ implies $\|v\| \leq N\|\omega\|$, for $v, \omega \in E$ and is called solid if $\operatorname{int} P \neq \emptyset$.

Definition 1.4 ([3]) Let $\left\{v_{m}\right\}$ be a sequence in a solid cone P. We say that $\left\{v_{m}\right\}$ is a $c$ sequence, if for any $c \in \mathrm{P}$ with $\theta \ll c$ there exists $m_{0} \in \mathbb{N}$ such that $v_{m} \ll c$ for all $m>m_{0}$.

Lemma 1.5 ([3]) If $\left\{v_{m}\right\}$ is a c-sequence in a solid cone $\mathrm{P}$ and $\kappa$ is arbitrary (but given) in $\mathrm{P}$, then $\left\{\kappa v_{m}\right\}$ is also a c-sequence.

Lemma 1.6 ([4]) On a real Banach algebra $\mathcal{E}$ with a solid cone $\mathcal{E}$, the following statements hold:

$1 \varsigma \ll \omega$ if $\varsigma \leq v \ll \omega$

$2 \varsigma=\theta$ if $\varsigma \ll \omega$ for every $\omega \gg \theta$.

Let $\mathcal{E}$ be a Banach algebra and $\mathrm{P} \subset \mathcal{E}$ be a cone. Then $(\mathrm{e}-v)$ is an invertible element in P for any $v \in$ P with $\rho(v)<1$.

Definition 1.7 ([23]) Suppose $\mathcal{E}$ is a Banach algebra with unit e and $\mathrm{P} \subseteq \mathcal{E}$ is a cone. $\mathrm{P}$ is called algebra cone if $\mathrm{e} \in \mathrm{P}$ and for $v, \omega \in \mathrm{P}, v \omega \in P$.

In what follows we consider that $\mathcal{E}\left(\mathcal{E}_{d}\right)$ represents a real (divisible) Banach algebra with a unit e and $\theta$ be its zero element, $\mathrm{P}$ is a solid cone in $\mathcal{E}, \mathrm{P}_{\mathcal{E}_{d}}$ a normal algebra cone in $\mathcal{E}_{d}$ with a normal constant $N$ and $\mathrm{X}$ is a non-empty set.

Definition 1.8 (see [24]) A mapping $d: X \times X \rightarrow \mathcal{E}$ is a cone metric on $\mathrm{X}$ if

(a) $0 \leq \mathrm{d}(v, \omega)$ for all $v, \omega \in \mathrm{X}$ and $\mathrm{d}(v, \omega)=0$ if and only if $v=\omega$,

(b) $\mathrm{d}(v, \omega)=\mathrm{d}(\omega, v)$ for all $v, \omega \in \mathrm{X}$,

(c) $\mathrm{d}(v, \omega) \leq \mathrm{d}(v, \eta)+\mathrm{d}(\eta, \omega)$, 
for all $v, \omega, \eta \in \mathrm{X}$. The pair $(\mathrm{X}, \mathrm{d})$ is said to be a cone metric space over Banach algebra, in short, CMS.

Definition 1.9 (see [25]) A mapping $\mathrm{q}: \mathrm{X} \times \mathrm{X} \rightarrow \mathcal{E}$ is said to be a quasi-cone metric if

(a) $0 \leq \mathrm{q}(v, \omega)$ for all $v, \omega \in \mathrm{X}$,

(b) $\mathrm{q}(v, \omega)=0=\mathrm{q}(\omega, v)$ if and only if $v \neq \omega$,

(c) $\mathrm{q}(v, \omega) \leq \mathrm{q}(v, \eta)+\mathrm{q}(\eta, \omega)$,

for all $v, \omega, \eta \in \mathrm{X}$. The triplet $(\mathrm{X}, \mathrm{q}, \mathcal{E})$ is said to be a quasi-cone metric space over Banach algebra, in short, qCMS.

A quasi-cone metric space is called $\Delta$-symmetric, if there exists an invertible element $\Delta \in \mathcal{E}$ such that

$$
\Delta^{-1} \mathbf{q}(v, \omega) \leq \mathrm{q}(\omega, v) \leq \Delta \mathrm{q}(v, \omega)
$$

for all $v, \omega \in \mathrm{X}$.

Definition 1.10 Suppose $(\mathrm{X}, \mathrm{q}, \mathcal{E})$ is a $q C M S, v \in \mathrm{X}$ and $\left\{v_{m}\right\}_{m \geq 1}$ is a sequence in $\mathrm{X}$. Then

(a) $\left\{v_{m}\right\}_{m \geq 1}$ (bi)-converges to $v$ if for $c \in \mathcal{E}$ with $\theta \ll c$, there is a natural number $N$ satisfying $\mathrm{q}\left(v_{m}, v\right) \ll c$ and $\mathrm{q}\left(v, v_{m}\right) \ll c$ for $m \geq N$. We denote $\lim _{m \rightarrow \infty} v_{m}=v$ or $v_{m} \rightarrow v$.

(b) $\left\{v_{m}\right\}_{m \geq 1}$ is a (l)(left)-Cauchy ((r)(right)-Cauchy)) if for $c \in \mathcal{E}$ with $\theta \ll c$, there exists a natural number $N$ satisfying $\mathbf{q}\left(v_{m}, v_{p}\right) \ll c$ (respectively, $\mathbf{q}\left(v_{p}, v_{m}\right) \ll c$ for $m>p \geq N$.

(c) $\left\{v_{m}\right\}_{m \geq 1}$ is a bi-Cauchy if for $c \in \mathcal{E}$ with $\theta \ll c$, there exists a natural number $N$ satisfying $\mathrm{q}\left(v_{m}, v_{p}\right) \ll c$ for $m, p \geq N$.

(d) $(\mathrm{X}, \mathrm{q}, \mathcal{E})$ is $(\mathrm{l})$-complete $((\mathrm{r})$-complete) if every (l)-Cauchy $((\mathrm{r})$-Cauchy) sequence is (bi)-convergent and is complete if it is (l) and (r)-complete.

Definition 1.11 We say that the mapping $\psi: \mathrm{P}_{\mathcal{E}_{d}} \rightarrow \mathrm{P}_{\mathcal{E}_{d}}$ is a $\psi$-operator if

(a) $\psi$ is an increasing;

(b) $\psi$ is a continuous bijection and has an inverse mapping $\psi^{-1}$ which is also continuous and increasing;

(c) $\psi(v+\omega) \leq \psi(v)+\psi(\omega)$ for all $v, \omega \in \mathrm{P}_{\mathcal{E}_{d}}$;

(d) $\psi(v \omega)=\psi(v) \psi(\omega)$ for all $v, \omega \in \mathrm{P}_{\mathcal{E}_{d}}$.

Remark 1.12 By Definition 1.11, the part of (c), we can obtain $\psi^{-1}(v)+\psi^{-1}(\omega) \leq \psi^{-1}(v+$ $\omega)$ for all $v, \omega \in b \mathrm{P}_{\mathcal{E}_{d}}$. In fact, note that $\psi(v+\omega) \leq \psi(v)+\psi(\omega)$ for all $v, \omega \in \mathrm{P}_{\mathcal{E}_{d}}$ and $\psi^{-1}$ is also a continuous and increasing operator, then

$$
\psi^{-1}(\psi(v+\omega)) \leq \psi^{-1}(\psi(v)+\psi(\omega))
$$

which yields

$$
v+\omega \leq \psi^{-1}(\psi(v)+\psi(\omega))
$$

Hence,

$$
\psi^{-1}(\psi(v))+\psi^{-1}(\psi(\omega)) \leq \psi^{-1}(\psi(v)+\psi(\omega))
$$


Since $\psi: \mathrm{P}_{\mathcal{E}_{d}} \rightarrow \mathrm{P}_{\mathcal{E}_{d}}$ is a continuous bijection, thus $\psi^{-1}(v)+\psi^{-1}(\omega) \leq \psi^{-1}(v+\omega)$, for all $v, \omega \in \mathrm{P}_{\mathcal{E}_{d}}$.

Remark 1.13 By Definition 1.11, the part of (d), we can obtain $\psi^{-1}(v \omega)=\psi^{-1}(v) \psi^{-1}(\omega)$, for $v, \omega \in \mathrm{P}_{\mathcal{E}_{d}}$.

Indeed, from $\psi(v \omega)=\psi(v) \psi(\omega)$ for $v, \omega \in \mathrm{P}_{\mathcal{E}}$ and $\psi^{-1}: \mathrm{P}_{\mathcal{E}} \rightarrow \mathrm{P}_{\mathcal{E}}$ is also continuous, we get

$$
\psi^{-1}(\psi(v \omega))=\psi^{-1}(\psi(v) \psi(\omega))
$$

which yields

$$
v \omega=\psi^{-1}(\psi(v) \psi(\omega)) .
$$

Then we obtain

$$
\psi^{-1}(\psi(v)) \psi^{-1}(\psi(\omega))=\psi^{-1}(\psi(v) \psi(\omega))
$$

Thanks to that $\psi: \mathrm{P}_{\mathcal{E}} \rightarrow \mathrm{P}_{\mathcal{E}}$ is a continuous bijection, $\psi^{-1}(v \omega)=\psi^{-1}(v) \psi^{-1}(\omega)$, for all $v, \omega \in \mathrm{P}_{\mathcal{E}_{d}}$.

Remark 1.14 For example, let $E_{d}=\mathrm{R}$ be a divisible Banach algebra, $\mathrm{P} \mathcal{E}_{d}=\{v \in \mathcal{E} \mid v \geq 0\}$ be a normal cone in $\mathcal{E}_{d}$, suppose $\psi: \mathrm{P}_{\mathcal{E}_{d}} \rightarrow \mathrm{P}_{\mathcal{E}_{d}}$, defined by $\psi(v)=v^{\frac{1}{5}}$ and then $\psi^{-1}(v)=v^{5}$, for all $v \in \mathrm{P}_{\mathcal{E}_{d}}$.

\section{Main results}

Lemma 2.1 Let (X, q, $\left.\mathcal{E}_{d}\right)$ be a $\Delta$-symmetric $q C M S$ over a divisible Banach algebra, $\left\{v_{m}\right\}$ a sequence in $\mathrm{X}$. If there exists $\kappa \in \mathrm{P}_{\mathcal{E}_{d}}$, with $\rho(\kappa)<1$ such that

$$
\mathrm{q}\left(v_{m}, v_{m+1}\right) \leq \kappa \mathrm{q}\left(v_{m-1}, v_{m}\right)
$$

for all $m \in \mathbb{N}$, then $\left\{v_{m}\right\}$ is a (bi)-Cauchy sequence.

Proof First of all, we remark that, successively applying Eq. (2), we have

$$
\mathbf{q}\left(v_{m+1}, v_{m}\right) \leq \kappa^{m} \mathbf{q}\left(v_{1}, v_{0}\right) .
$$

Let $m>p \geq N$. Thereupon,

$$
\begin{aligned}
\mathrm{q}\left(v_{m}, v_{p}\right) & \leq \mathrm{q}\left(v_{m}, v_{m-1}\right)+\mathrm{q}\left(v_{m-1}, v_{m-2}\right)+\cdots+\mathrm{q}\left(v_{p+1}, v_{p}\right) \\
& \leq\left(\kappa^{m-1}+\kappa^{m-2}+\cdots+\kappa^{p}\right) \mathrm{q}\left(v_{1}, v_{0}\right) \\
& \leq \kappa^{p} \sum_{j=0}^{m-p-1} \kappa^{j} \mathrm{q}\left(v_{1}, v_{0}\right) \\
& \leq \kappa^{p} \sum_{j=0}^{\infty} \kappa^{j} \mathrm{q}\left(v_{1}, v_{0}\right) .
\end{aligned}
$$


Now, since $\rho(\kappa)<1$, and taking into account Proposition 1.2, we see that $(\mathrm{e}-\kappa)$ is an invertible element and $(\mathrm{e}-\kappa)^{-1}=\sum_{j=0}^{\infty} \kappa^{j}$ and the above inequality becomes

$$
\mathrm{q}\left(v_{m}, v_{p}\right) \leq \kappa^{p}(\mathrm{e}-\kappa)^{-1} \mathbf{q}\left(v_{1}, v_{0}\right) .
$$

For a given $c$, with $\theta \ll c$, we choose $\delta>0$ such that $c+N_{\delta}(\theta) \subseteq \mathrm{P}_{\mathcal{E}_{d}}$. (Here $N_{\delta}(\theta)=\{\omega \in$ $\left.\left.\mathcal{E}_{d}:\|\omega\|\right\}<\delta\right\}$.) Letting $p_{0} \in \mathbb{N}$ such that $\kappa^{p} \mathrm{q}\left(v_{1}, v_{0}\right) \in N_{\delta}(\theta)$ for all $p \geq p_{0}$ we get $\kappa^{p}(\mathrm{e}-$ $\kappa)^{-1} \mathrm{q}\left(v_{1}, v_{0}\right) \ll c$, for all $p \geq p_{0}$. Therefore,

$$
\mathrm{q}\left(v_{m}, v_{p}\right) \leq \kappa^{p}(\mathrm{e}-\kappa)^{-1} \mathrm{q}\left(v_{1}, v_{0}\right) \ll c, \quad \text { for all } m>p .
$$

Then by (b) in Definition 1.10 it follows that the sequence $\left\{v_{m}\right\}$ is (l)-Cauchy. On the other hand, from Definition 1.4, we see that the sequence $\left\{q\left(v_{m}, v_{p}\right)\right\}$ is $c$-convergent and moreover in view of Lemma 1.5 the sequence $\left\{\Delta \mathrm{q}\left(v_{m}, v_{p}\right)\right\}$, where $\Delta \in \mathrm{P}_{\mathcal{E}_{d}}$, is also a $c$-sequence, that is,

$$
\Delta \mathrm{q}\left(v_{m}, v_{p}\right) \ll c,
$$

for all $m>p>p_{0}$. But, since the space $\left(\mathrm{X}, \mathrm{q}, \mathcal{E}_{d}\right)$ is supposed to be $\Delta$-symmetric, we have

$$
\mathrm{q}\left(v_{p}, v_{m}\right) \leq \Delta \mathrm{q}\left(v_{m}, v_{p}\right)
$$

and taking Lemma 1.5 into account we get $\mathrm{q}\left(v_{p}, v_{m}\right) \ll c$, for all $m>p \geq p_{0}$, which means the sequence $\left\{v_{m}\right\}$ is (r)-Cauchy. Obviously, in view of statement $(c)$ in Definition 1.10, it follows that $\left\{v_{m}\right\}$ is a (bi)-Cauchy sequence.

Let $\left(\mathcal{E}_{d}\right)$ be a real (divisible) Banach algebra with a unit e and $\theta$ be its zero element and $\mathrm{P}_{\mathcal{E}_{d}}^{1}$ be a normal algebra cone with constant $N=1$ in $\mathcal{E}_{d}$.

Theorem 2.2 Let $\left(\mathrm{X}, \mathrm{q}, \mathcal{E}_{d}\right)$ be a complete $\Delta$-symmetric $q C M S$ over $\mathcal{E}_{d}$ and $\mathrm{P}_{\mathcal{E}_{d}}^{1}$. Suppose that $\psi: \mathrm{P}_{\mathcal{E}_{d}}^{1} \rightarrow \mathrm{P}_{\mathcal{E}_{d}}^{1}$ is a $\psi$-operator and $\mathcal{U}, \mathcal{V}: \mathrm{X} \rightarrow \mathrm{X}$ are mappings satisfying the conditions

$$
\begin{aligned}
& \psi(\mathrm{q}(v, \mathcal{U} v))+\psi(\mathrm{q}(\omega, \mathcal{V} \omega)) \leq k \psi(\mathrm{q}(v, \omega)), \\
& \psi(\mathrm{q}(v, \mathcal{V} v))+\psi(\mathrm{q}(\omega, \mathcal{U} \omega)) \leq k \psi(\mathrm{q}(v, \omega)),
\end{aligned}
$$

for all $v, \omega \in \mathrm{X}$ with $\mathrm{q}(v, \omega)>\theta$, where $\psi(e) \leq k<\psi(2 e)$ in $\mathrm{P}_{\mathcal{E}_{d}^{1}}$. Then $\mathcal{U}$ and $\mathcal{V}$ have a common fixed point.

Proof Let $v_{0} \in D$ be an arbitrary point and the sequence $\left\{v_{m}\right\}$ defined by

$$
v_{2 m+1}=\mathcal{U} v_{2 m}, \quad v_{2 m+2}=\mathcal{V} v_{2 m+1}, \quad \text { for } m \in \mathbb{N} .
$$

Then, setting $v=v_{2 m}$ and $\omega=v_{2 m+1}$, we get

$$
\psi\left(\mathrm{q}\left(\tau_{2 m}, \tau_{2 m+1}\right)\right)+\psi\left(\mathrm{q}\left(\tau_{2 m+1}, v_{2 m+2}\right)\right)
$$




$$
\begin{aligned}
& =\psi\left(\mathrm{q}\left(\tau_{2 m}, \mathcal{U} \tau_{2 m}\right)\right)+\psi\left(\mathrm{q}\left(\tau_{2 m+1}, \mathcal{V} \tau_{2 m+1}\right)\right) \\
& \leq k \psi\left(\mathrm{q}\left(\tau_{2 m}, \tau_{2 m+1}\right)\right)
\end{aligned}
$$

and then

$$
\psi\left(\mathrm{q}\left(\tau_{2 m}, v_{2 m+1}\right)\right)+\psi\left(\mathrm{q}\left(v_{2 m+1}, v_{2 m+2}\right)\right) \leq k \psi\left(\mathrm{q}\left(v_{2 m}, v_{2 m+1}\right)\right) .
$$

Also if we put $v=v_{2 m-1}$ and $\omega=v_{2 m}$, then we have

$$
\begin{aligned}
\psi & \left(\mathrm{q}\left(\tau_{2 m-1}, v_{2 m}\right)\right)+\psi\left(\mathrm{q}\left(\tau_{2 m}, \tau_{2 m+1}\right)\right) \\
& =\psi\left(\mathrm{q}\left(\tau_{2 m-1}, \mathcal{V} \tau_{2 m-1}\right)\right)+\psi\left(\mathrm{q}\left(v_{2 m}, \mathcal{U} \tau_{2 m}\right)\right) \\
& \leq k \psi\left(\mathrm{q}\left(v_{2 m-1}, v_{2 m}\right)\right) .
\end{aligned}
$$

Thus,

$$
\psi\left(\mathrm{q}\left(v_{2 m-1}, v_{2 m}\right)\right)+\psi\left(\mathrm{q}\left(v_{2 m}, v_{2 m+1}\right)\right) \leq k \psi\left(\mathrm{q}\left(v_{2 m-1}, v_{2 m}\right)\right) .
$$

Moreover, applying $\psi^{-1}$ in (8), (9) and keeping in mind the properties of the operator $\psi^{-1}$, it follows

$$
\begin{aligned}
& \mathbf{q}\left(\tau_{2 m}, \tau_{2 m+1}\right)+\mathbf{q}\left(\tau_{2 m+1}, \tau_{2 m+2}\right) \leq \psi^{-1}(k) \mathbf{q}\left(v_{2 m}, \tau_{2 m+1}\right), \\
& \mathbf{q}\left(\tau_{2 m-1}, v_{2 m}\right)+\mathbf{q}\left(\tau_{2 m}, \tau_{2 m+1}\right) \leq \psi^{-1}(k) \mathbf{q}\left(\tau_{2 m-1}, v_{2 m}\right),
\end{aligned}
$$

or by simplifying, we obtain

$$
\begin{aligned}
& \mathbf{q}\left(v_{2 m+1}, v_{2 m+2}\right) \leq\left(\psi^{-1}(k)-\mathrm{e}\right) \mathbf{q}\left(v_{2 m}, v_{2 m+1}\right) \\
& \mathbf{q}\left(v_{2 m}, v_{2 m+1}\right) \leq\left(\psi^{-1}(k)-\mathrm{e}\right) \mathbf{q}\left(v_{2 m-1}, v_{2 m}\right) .
\end{aligned}
$$

Denoting $\kappa=\psi^{-1}(k)-\mathrm{e}$, the above inequalities pass into

$$
\mathrm{q}\left(v_{m}, v_{m+1}\right) \leq \kappa \mathrm{q}\left(v_{m-1}, v_{m}\right),
$$

for any positive integer $m$. Now, by hypothesis $\psi(\mathrm{e}) \leq k<\psi(2 \mathrm{e})$ it follows that $\theta \leq$ $\psi^{-1}(k)-\mathrm{e}<\mathrm{e}$ and since the cone $\mathrm{P}_{\mathcal{E}_{d}}$ is normal (with $N=1$ ),

$$
\|\kappa\| \leq\|\mathrm{e}\|=1
$$

and then $\rho(\kappa)<1$. Thereupon, by Lemma 2.1 we see that the sequence $\left\{v_{m}\right\}$ is (bi)-Cauchy. Further, we can find $v_{*} \in \mathrm{X}$ such that the sequence $\left\{v_{m}\right\}$ converges to $v_{*}$. That is, for every $c \gg \theta$ there exists $m_{1} \in \mathbb{N}$ such that $\mathrm{q}\left(v_{2 m}, v_{*}\right) \ll c$ and $\mathrm{q}\left(v_{2 m-1}, v_{*}\right) \ll c$, for $m \geq m_{1}$. Thus, replacing in (5) $v$ by $v_{2 m}$ and $\omega$ by $v_{*}$ we have

$$
\psi\left(\mathrm{q}\left(v_{2 m}, \mathcal{U} v_{2 m}\right)\right)+\psi\left(\mathrm{q}\left(v_{*}, \mathcal{V} v_{*}\right)\right) \leq k \psi\left(\mathrm{q}\left(v_{2 m}, v_{*}\right)\right)
$$


and from (c), Definition 1.11,

$$
\psi\left(\mathrm{q}\left(v_{2 m}, \mathcal{U} v_{2 m}\right)+\mathrm{q}\left(v_{*}, \mathcal{V} v_{*}\right)\right) \leq k \psi\left(\mathrm{q}\left(v_{2 m}, v_{*}\right)\right) .
$$

Moreover, by Definition 1.9 and Remark 1.13, we have

$$
\begin{aligned}
\mathrm{q}\left(v_{*}, \mathcal{V}_{v_{*}}\right) & \leq \mathrm{q}\left(v_{2 m}, \mathcal{U} v_{2 m}\right)+\mathrm{q}\left(v_{*}, \mathcal{V}_{v_{*}}\right) \leq \psi^{-1}(k) \mathrm{q}\left(\tau_{2 m}, v_{*}\right) \\
& \ll \psi^{-1}(k) \mathcal{c}
\end{aligned}
$$

and from Lemma 1.6 we obtain $\mathrm{q}\left(v_{*}, \mathcal{V} v_{*}\right)=\theta$. Therefore, $\mathcal{V}_{v_{*}}=v_{*}$. Similarly, choosing in (6) $v=v_{2 m-1}$ and $\omega=v_{*}$, and taking into account the properties of $\psi$ we have

$$
\begin{aligned}
\psi\left(\mathrm{q}\left(v_{2 m-1}, \mathcal{V} v_{2 m-1}\right)+\mathrm{q}\left(v_{*}, \mathcal{U} v_{*}\right)\right) & \leq \psi\left(\mathrm{q}\left(v_{2 m-1}, \mathcal{V} v_{2 m-1}\right)\right)+\psi\left(\mathrm{q}\left(v_{*}, \mathcal{U} v_{*}\right)\right) \\
& \leq k \psi\left(\mathrm{q}\left(v_{2 m-1}, v_{*}\right)\right),
\end{aligned}
$$

which leads us to

$$
\begin{aligned}
\mathrm{q}\left(v_{*}, \mathcal{U} v_{*}\right) & \leq \mathrm{q}\left(v_{2 m-1}, \mathcal{V}_{\tau_{2 m-1}}\right)+\mathrm{q}\left(v_{*}, \mathcal{U} v_{*}\right) \leq \psi^{-1}(k) \mathrm{q}\left(\tau_{2 m-1}, v_{*}\right) \\
& \ll \psi^{-1}(k) c .
\end{aligned}
$$

Consequently, $\mathcal{U} v_{*}=v_{*}=\mathcal{V}_{v_{*}}$.

Example 2.3 Let $\mathcal{E}_{d}=\mathbb{R}^{2}, \mathrm{P}_{\mathcal{E}}^{1}=\{(v, \omega): v, \omega \geq 0\}$ and for any $\left(\tau_{1}, \omega_{1}\right),\left(\tau_{2}, \omega_{2}\right) \in \mathcal{E}_{d}$ we define the multiplication as $\left(v_{1}, \omega_{1}\right)\left(v_{2}, \omega_{2}\right)=\left(v_{1} \tau_{2}, \omega_{1} \omega_{2}\right)$. Then $\mathcal{E}_{d}$ is a Banach algebra with a unit $\mathrm{e}=(1,1)$. Let $\mathrm{X}=\{1,3,4,5\}$ and $\mathrm{q}: \mathrm{X} \times \mathrm{X} \rightarrow \mathcal{E}$ defined by

$$
q(v, \omega)= \begin{cases}\left(v-\omega, \frac{v-\omega}{2}\right) & \text { if } v \geq \omega \\ (2(v-\omega), v-\omega) & \text { if } v<\omega\end{cases}
$$

be a 2-symmetric quasi-metric on $\mathrm{X}$. Consider also the mappings $\mathcal{U}, \mathcal{V}: \mathrm{X} \rightarrow \mathrm{X}$ defined by $\mathcal{U} 1=1, \mathcal{U} 3=3, \mathcal{U} 4=3, \mathcal{U} 5=5$ and $\mathcal{V} 1=1, \mathcal{V} 3=3, \mathcal{V} 4=4, \mathcal{V} 5=4$. Then we have

$$
\begin{aligned}
& q(1,3)=(4,2), \quad q(1,4)=(6,3), \quad q(1,5)=(8,4), \quad q(3,4)=(2,1), \\
& q(3,5)=(4,2), \quad q(4,5)=(2,1), \quad q(1, \mathcal{U} 1)=(0,0), \quad q(3, \mathcal{U} 3)=(0,0), \\
& q(4, \mathcal{U} 4)=\left(1, \frac{1}{2}\right), \quad q(5, \mathcal{U} 5)=(0,0), \quad q(1, \mathcal{V} 1)=(0,0), \\
& q(3, \mathcal{V} 3)=(0,0), \quad q(4, \mathcal{V} 4)=(0,0), \quad q(5, \mathcal{V} 5)=\left(1, \frac{1}{2}\right) .
\end{aligned}
$$

Let $\psi: \mathrm{P}^{1} \mathcal{E} \rightarrow \mathrm{P}^{1} \mathcal{E}, \psi((v, \omega))=(\sqrt[3]{v}, \sqrt[3]{\omega})$ and $k=\left(\frac{9}{8}, \frac{9}{8}\right)$.

Therefore:

$1 v=1, \omega=3$

$$
\psi(\mathrm{q}(1, \mathcal{U} 1))+\psi(\mathrm{q}(3, \mathcal{V} 3))=(0,0) \leq\left(\frac{9}{2}, \frac{9}{4}\right)=k \psi(\mathrm{q}(1,3)),
$$




$$
\psi(\mathrm{q}(1, \mathcal{V} 1))+\psi(\mathrm{q}(3, \mathcal{U} 3))=(0,0) \leq\left(\frac{9}{2}, \frac{9}{4}\right)=k \psi(\mathrm{q}(1,3))
$$

$2 v=1, \omega=4$

$$
\begin{aligned}
& \psi(\mathrm{q}(1, \mathcal{U} 1))+\psi(\mathrm{q}(4, \mathcal{V} 4))=(0,0) \leq\left(\frac{27}{4}, \frac{27}{8}\right)=k \psi(\mathrm{q}(1,4)), \\
& \psi(\mathrm{q}(1, \mathcal{V} 1))+\psi(\mathrm{q}(4, \mathcal{U} 4))=\left(1, \frac{1}{2}\right) \leq\left(\frac{27}{4}, \frac{27}{8}\right)=k \psi(\mathrm{q}(1,4))
\end{aligned}
$$

$3 v=1, \omega=5$

$$
\begin{aligned}
& \psi(\mathrm{q}(1, \mathcal{U} 1))+\psi(\mathrm{q}(5, \mathcal{V} 5))=\left(1, \frac{1}{2}\right) \leq\left(9, \frac{9}{2}\right)=k \psi(\mathrm{q}(1,5)) \\
& \psi(\mathrm{q}(1, \mathcal{V} 1))+\psi(\mathrm{q}(5, \mathcal{U} 5))=(0,0) \leq\left(9, \frac{9}{2}\right)=k \psi(\mathrm{q}(1,5))
\end{aligned}
$$

4. $v=3, \omega=4$

$$
\begin{aligned}
& \psi(\mathrm{q}(3, \mathcal{U} 3))+\psi(\mathrm{q}(4, \mathcal{V} 4))=(0,0) \leq\left(\frac{9}{4}, \frac{9}{8}\right)=k \psi(\mathrm{q}(3,4)) \\
& \psi(\mathrm{q}(3, \mathcal{V} 3))+\psi(\mathrm{q}(4, \mathcal{U} 4))=\left(1, \frac{1}{2}\right) \leq\left(\frac{9}{4}, \frac{9}{8}\right)=k \psi(\mathrm{q}(3,4))
\end{aligned}
$$

$5 v=3, \omega=5$

$$
\begin{aligned}
& \psi(\mathrm{q}(3, \mathcal{U} 3))+\psi(\mathrm{q}(5, \mathcal{V} 5))=\left(1, \frac{1}{2}\right) \leq\left(\frac{9}{2}, \frac{9}{4}\right)=k \psi(\mathrm{q}(3,5)) \\
& \psi(\mathrm{q}(3, \mathcal{V} 3))+\psi(\mathrm{q}(5, \mathcal{U} 5))=(0,0) \leq\left(\frac{9}{2}, \frac{9}{4}\right)=k \psi(\mathrm{q}(3,5))
\end{aligned}
$$

$6 v=4, \omega=5$

$$
\begin{aligned}
& \psi(\mathrm{q}(4, \mathcal{U} 4))+\psi(\mathrm{q}(5, \mathcal{V} 5))=(2,1) \leq\left(\frac{9}{2}, \frac{9}{4}\right)=k \psi(\mathrm{q}(3,5)) \\
& \psi(\mathrm{q}(4, \mathcal{V} 4))+\psi(\mathrm{q}(5, \mathcal{U} 5))=(0,0) \leq\left(\frac{9}{2}, \frac{9}{4}\right)=k \psi(\mathrm{q}(3,5))
\end{aligned}
$$

Consequently, the assumptions of Theorem 2.2 are verified and the mappings $\mathcal{U}, \mathcal{V}$ have 2 common fixed points, these being $v=1, v=3$.

Corollary 2.4 Let $\left(\mathrm{X}, \mathrm{q}, \mathcal{E}_{d}\right)$ be a complete $\Delta$-symmetric $q C M S$ over $\mathcal{E}_{d}$ and $\mathrm{P}_{\mathcal{E}_{d}}^{1}$. Suppose that $\psi: \mathrm{P}_{\mathcal{E}_{d}}^{1} \rightarrow \mathrm{P}_{\mathcal{E}_{d}}^{1}$ is a $\psi$-operator and $\mathcal{U}: \mathrm{X} \rightarrow \mathrm{X}$ is a mapping satisfying the condition

$$
\psi(\mathbf{q}(v, \mathcal{U} v))+\psi(\mathbf{q}(\omega, \mathcal{U} \omega)) \leq k \psi(\mathbf{q}(v, \omega))
$$

for all $v, \omega \in \mathrm{X}$ with $\mathrm{q}(v, \omega)>\theta$, where $\psi(e) \leq k<\psi(2 e)$ in $\mathrm{P}_{\mathcal{E}_{d}^{1}}$. Then $\mathcal{U}$ has a fixed point. Proof Put $\mathcal{U}=\mathcal{V}$ in Theorem 2.2. 
Theorem 2.5 Let $\left(\mathrm{X}, \mathrm{q}, \mathcal{E}_{d}\right)$ be a complete $\Delta$-symmetric $q C M S$ over $\mathcal{E}_{d}$ and $\mathrm{P}_{\mathcal{E}_{d}}^{1}$. Suppose that $\psi: \mathrm{P}_{\mathcal{E}_{d}}^{1} \rightarrow \mathrm{P}_{\mathcal{E}_{d}}^{1}$ is a $\psi$-operator and $\mathcal{U}, \mathcal{V}: \mathrm{X} \rightarrow \mathrm{X}$ are mappings satisfying the conditions

$$
\begin{aligned}
& \alpha_{1} \psi\left(\mathbf{q}(\mathcal{U} v, \mathcal{V} \omega)+\alpha_{2} \psi(\mathbf{q}(v, \mathcal{U} v))+\alpha_{3} \psi(\mathrm{q}(\omega, \mathcal{V} \omega)) \leq \beta \psi(\mathrm{q}(v, \omega))\right. \\
& \alpha_{1} \psi\left(\mathbf{q}(\mathcal{V} v, \mathcal{U} \omega)+\alpha_{2} \psi(\mathbf{q}(v, \mathcal{V} v))+\alpha_{3} \psi(\mathbf{q}(\omega, \mathcal{U} \omega)) \leq \beta \psi(\mathbf{q}(v, \omega))\right.
\end{aligned}
$$

for all $v, \omega \in \mathrm{X}$ with $\mathrm{q}(v, \omega)>\theta$, where

$$
\theta<\psi^{-1}\left(\alpha_{2}\right), \quad \theta \leq \psi^{-1}(\beta)<\psi^{-1}\left(\alpha_{1}\right)+\psi^{-1}\left(\alpha_{2}\right)+\psi^{-1}\left(\alpha_{3}\right),
$$

in $\mathrm{P}_{\mathcal{E}_{d}}^{1}$. Then $\mathcal{U}$ and $\mathcal{V}$ have a common fixed point. Moreover, if $\psi^{-1}(\beta)<\psi^{-1}\left(\alpha_{1}\right)$ then the common fixed point is unique.

Proof Let $\left\{v_{m}\right\}$ be the sequence in X defined by (7). Letting $v=v_{2 m}$ and $\omega=v_{2 m+1}$ in (11) we have

$$
\begin{aligned}
& \alpha_{1} \psi\left(\mathbf{q}\left(\mathcal{U} \tau_{2 m}, \mathcal{V} \tau_{2 m+1}\right)\right)+\alpha_{2} \psi\left(\mathrm{q}\left(\tau_{2 m}, \mathcal{U} \tau_{2 m}\right)\right)+\alpha_{3} \psi\left(\mathrm{q}\left(\tau_{2 m+1}, \mathcal{V}_{\tau_{2 m+1}}\right)\right) \\
& \quad \leq \beta \psi\left(\mathrm{q}\left(\tau_{2 m}, \tau_{2 m+1}\right)\right)
\end{aligned}
$$

or

$$
\begin{aligned}
& \alpha_{1} \psi\left(\mathbf{q}\left(v_{2 m+1}, v_{2 m+2}\right)\right)+\alpha_{2} \psi\left(\mathbf{q}\left(\tau_{2 m}, v_{2 m+1}\right)\right)+\alpha_{3} \psi\left(\mathbf{q}\left(v_{2 m+1}, v_{2 m+2}\right)\right) \\
& \quad \leq \beta \psi\left(\mathbf{q}\left(v_{2 m}, v_{2 m+1}\right)\right) .
\end{aligned}
$$

Taking into account the properties of $\psi^{-1}$, we have

$$
\begin{aligned}
& \psi^{-1}\left(\alpha_{1}\right) \mathrm{q}\left(\tau_{2 m+1}, \tau_{2 m+2}\right)+\psi^{-1}\left(\alpha_{2}\right) \mathrm{q}\left(\tau_{2 m}, \tau_{2 m+1}\right)+\psi^{-1}\left(\alpha_{3}\right) \alpha_{3} \mathrm{q}\left(\tau_{2 m+1}, v_{2 m+2}\right) \\
& \quad \leq \psi^{-1}\left[\alpha _ { 1 } \psi \left(\mathrm{q}\left(\tau_{2 m+1}, \tau_{2 m+2}\right)+\alpha_{2} \psi\left(\mathrm{q}\left(\tau_{2 m}, v_{2 m+1}\right)\right)+\alpha_{3} \psi\left(\mathrm{q}\left(\tau_{2 m+1}, v_{2 m+2}\right)\right)\right.\right. \\
& \leq \psi^{-1}\left[\beta \psi\left(\mathrm{q}\left(\tau_{2 m}, \tau_{2 m+1}\right)\right)\right]=\psi^{-1}(\beta) \mathrm{q}\left(\tau_{2 m}, \tau_{2 m+1}\right)
\end{aligned}
$$

and moreover

$$
\left(\psi^{-1}\left(\alpha_{1}\right)+\psi^{-1}\left(\alpha_{3}\right)\right) q\left(v_{2 m+1}, v_{2 m+2}\right) \leq\left(\psi^{-1}(\beta)-\psi^{-1}\left(\alpha_{2}\right)\right) q\left(v_{2 m}, v_{2 m+1}\right) .
$$

Therefore, since the Banach algebra is divisible, we get

$$
\mathrm{q}\left(\tau_{2 m+1}, v_{2 m+2}\right) \leq\left(\psi^{-1}\left(\alpha_{1}\right)+\psi^{-1}\left(\alpha_{3}\right)\right)^{-1}\left(\psi^{-1}(\beta)-\psi^{-1}\left(\alpha_{2}\right)\right) \mathrm{q}\left(v_{2 m}, v_{2 m+1}\right) .
$$

If we denote $\kappa=\left(\psi^{-1}\left(\alpha_{1}\right)+\psi^{-1}\left(\alpha_{3}\right)\right)^{-1}\left(\psi^{-1}(\beta)-\psi^{-1}\left(\alpha_{2}\right)\right)$, we can easily see that $\theta \leq \kappa<\mathrm{e}$ and

$$
\mathrm{q}\left(\tau_{2 m+1}, \tau_{2 m+2}\right) \leq \kappa \mathrm{q}\left(\tau_{2 m}, \tau_{2 m+1}\right) .
$$


In the same way, for $v=v_{2 m-1}$ and $\omega=v_{2 m}$, (12) becomes

$$
\begin{aligned}
& \alpha_{1} \psi\left(\mathrm{q}\left(\mathcal{V}_{\tau_{2 m-1}}, \mathcal{U} \tau_{2 m}\right)\right)+\alpha_{2} \psi\left(\mathrm{q}\left(\tau_{2 m-1}, \mathcal{V} \tau_{2 m-1}\right)\right)+\alpha_{3} \psi\left(\mathrm{q}\left(\tau_{2 m}, \mathcal{U} \tau_{2 m}\right)\right) \\
& \quad \leq \beta \psi\left(\mathrm{q}\left(v_{2 m-1}, \tau_{2 m}\right)\right)
\end{aligned}
$$

or, equivalent

$$
\alpha_{1} \psi\left(\mathrm{q}\left(\tau_{2 m}, \tau_{2 m+1}\right)\right)+\alpha_{2} \psi\left(\mathrm{q}\left(\tau_{2 m-1}, \tau_{2 m}\right)\right)+\alpha_{3} \psi\left(\mathrm{q}\left(\tau_{2 m}, \tau_{2 m+1}\right)\right) \leq \beta \psi\left(\mathrm{q}\left(\tau_{2 m-1}, \tau_{2 m}\right)\right) .
$$

Thereupon,

$$
\left(\psi^{-1}\left(\alpha_{1}\right)+\psi^{-1}\left(\alpha_{3}\right)\right) q\left(\tau_{2 m}, \tau_{2 m+1}\right) \leq\left(\psi^{-1}(\beta)-\psi^{-1}\left(\alpha_{2}\right)\right) \mathbf{q}\left(\tau_{2 m-1}, \tau_{2 m}\right),
$$

which yields

$$
\begin{aligned}
\mathrm{q}\left(\tau_{2 m}, \tau_{2 m+1}\right) & \leq\left(\psi^{-1}\left(\alpha_{1}\right)+\psi^{-1}\left(\alpha_{3}\right)\right)^{-1}\left(\psi^{-1}(\beta)-\psi^{-1}\left(\alpha_{2}\right)\right) \mathrm{q}\left(\tau_{2 m-1}, v_{2 m}\right) \\
& =\kappa \mathrm{q}\left(v_{2 m-1}, v_{2 m}\right)
\end{aligned}
$$

(here we took into account that the Banach algebra is divisible). Now, by (13) and (14) we have

$$
\mathrm{q}\left(v_{m}, v_{m+1}\right) \leq \kappa \mathrm{q}\left(v_{m-1}, v_{m}\right)
$$

for all $m \in \mathbb{N}$, where $\theta \leq \kappa<\mathrm{e}$. Then, by using Lemma 2.1, we see that the sequence $\left\{v_{m}\right\}$ is (bi)-Cauchy and since the $q C M S\left(\mathrm{X}, \mathrm{q}, \mathcal{E}_{d}\right)$ is complete, we can have $v_{*} \in \mathrm{X}$ such that $\left\{v_{m}\right\}$ converges to $v_{*}$. Thus, there exists $m_{2} \in \mathbb{N}$ such that for any $c \gg \theta$ we have $\mathrm{q}\left(v_{2 m}, v_{*}\right) \ll c$, $\mathrm{q}\left(v_{2 m-1}, v_{*}\right) \ll c$ and also $\mathrm{q}\left(v_{2 m}, v_{2 m+1}\right) \ll c, \mathrm{q}\left(\tau_{2 m+1}, v_{2 m+2}\right) \ll c$, for any $m \geq m_{1}$. Hence, by (11), respectively, (12) we have

$$
\begin{aligned}
\alpha_{3} \psi\left(\mathrm{q}\left(v_{*}, \mathcal{V} v_{*}\right)\right) \leq & \left.\alpha_{1} \psi\left(\mathrm{q}\left(\mathcal{U} v_{2 m}\right), \mathcal{V} v_{*}\right)\right)+\alpha_{2} \psi\left(\mathrm{q}\left(v_{2 m}, \mathcal{U} v_{2 m}\right)\right) \\
& +\alpha_{3} \psi\left(\mathrm{q}\left(v_{*}, \mathcal{V} v_{*}\right)\right) \leq \beta \psi\left(\mathrm{q}\left(\tau_{2 m}, v_{*}\right)\right), \\
\alpha_{3} \psi\left(\mathrm{q}\left(v_{*}, \mathcal{U} v_{*}\right)\right) \leq & \alpha_{1} \psi\left(\mathbf{q}\left(\mathcal{V}_{v_{2 m-1}}, \mathcal{U} v_{*}\right)\right)+\alpha_{2} \psi\left(\mathrm{q}\left(v_{2 m-1}, \mathcal{V}_{v_{2 m-1}}\right)\right) \\
& +\alpha_{3} \psi\left(\mathrm{q}\left(v_{*}, \mathcal{U} v_{*}\right)\right) \leq \beta \psi\left(\mathrm{q}\left(v_{2 m-1}, v_{*}\right)\right),
\end{aligned}
$$

for $m \geq m_{2}$. Moreover, applying $\psi^{-1}$ in the above inequalities,

$$
\begin{aligned}
& \psi^{-1}\left(\alpha_{3}\right) \mathbf{q}\left(v_{*}, \mathcal{V} v_{*}\right) \leq \psi^{-1}(\beta) \mathbf{q}\left(v_{2 m}, v_{*}\right), \\
& \psi^{-1}\left(\alpha_{3}\right) \mathbf{q}\left(v_{*}, \mathcal{U} v_{*}\right) \leq \psi^{-1}(\beta) \mathbf{q}\left(v_{2 m-1}\right),
\end{aligned}
$$

which are equivalent (since the Banach algebra is divisible) with

$$
\begin{aligned}
& \mathrm{q}\left(v_{*}, \mathcal{V} v_{*}\right) \leq\left(\psi^{-1}\left(\alpha_{2}\right)\right)^{-1} \psi^{-1}(\beta) \mathrm{q}\left(v_{2 m}, v_{*}\right) \ll\left(\psi^{-1}\left(\alpha_{2}\right)\right)^{-1} \psi^{-1}(\beta) c, \\
& \mathrm{q}\left(v_{*}, \mathcal{U} v_{*}\right) \leq\left(\psi^{-1}\left(\alpha_{3}\right)\right)^{-1} \psi^{-1}(\beta) \mathrm{q}\left(v_{2 m-1}, v_{*}\right) \ll\left(\psi^{-1}\left(\alpha_{2}\right)\right)^{-1} \psi^{-1}(\beta) c,
\end{aligned}
$$


for all $m \geq m_{2}$ and any $c \gg \theta$. Therefore, by Lemma 1.6, it follows that $\mathrm{q}\left(v_{*}, \mathcal{V}_{v_{*}}\right)=v_{*}$ and also $\mathrm{q}\left(v_{*}, \mathcal{V}_{*}\right)=v_{*}$, which means that $v_{*}$ is a common fixed point of the mappings $\mathcal{V}, \mathcal{U}$.

Finally, considering the additional hypothesis, we will prove the uniqueness of the common fixed point. Supposing, on the contrary, that there exists another point, let us say $\omega_{*} \in \mathrm{X}$ different from $v_{*}$, such that $\mathcal{V} v_{*}=v_{*}=\mathcal{U} v_{*}$, we have, by (11), for example,

$$
\alpha_{1} \psi\left(\mathbf{q}\left(\mathcal{U} v_{*}, \mathcal{V} \omega_{*}\right)\right)+\alpha_{2} \psi\left(\mathbf{q}\left(v_{*}, \mathcal{U} v_{*}\right)\right)+\alpha_{3} \psi\left(\mathbf{q}\left(\omega_{*}, \mathcal{V} \omega_{*}\right)\right) \leq \beta \psi\left(\mathbf{q}\left(v_{*}, \omega_{*}\right)\right)
$$

Thus,

$$
\begin{aligned}
& \psi^{-1}\left(\alpha_{1}\right) \mathbf{q}\left(v_{*}, \omega_{*}\right)+\psi^{-1}\left(\alpha_{2}\right) \mathbf{q}\left(v_{*}, v_{*}\right)+\psi^{-1}\left(\alpha_{3}\right) \mathbf{q}\left(\omega_{*}, \omega_{*}\right) \\
& \quad \leq \psi^{-1}\left(\alpha_{1} \psi\left(\mathbf{q}\left(\mathcal{U} v_{*}, \mathcal{V} \omega_{*}\right)\right)+\alpha_{2} \psi\left(\mathbf{q}\left(v_{*}, \mathcal{U} v_{*}\right)\right)+\alpha_{3} \psi\left(\mathbf{q}\left(\omega_{*}, \mathcal{V} \omega_{*}\right)\right)\right) \\
& \quad \leq \psi^{-1}\left(\beta \psi\left(\mathbf{q}\left(v_{*}, \omega_{*}\right)\right)\right) \\
& \quad=\psi^{-1}(\beta) \mathbf{q}\left(v_{*}, \omega_{*}\right),
\end{aligned}
$$

and we obtain

$$
\begin{aligned}
\mathrm{q}\left(v_{*}, \omega_{*}\right) & \leq\left(\psi^{-1}\left(\alpha_{1}\right)\right)^{-1} \psi^{-1}(\beta) \mathrm{q}\left(v_{*}, \omega_{*}\right) \leq\left[\left(\psi^{-1}\left(\alpha_{1}\right)\right)^{-1} \psi^{-1}(\beta)\right]^{2} \mathbf{q}\left(v_{*}, \omega_{*}\right) \\
& \leq\left[\left(\psi^{-1}\left(\alpha_{1}\right)\right)^{-1} \psi^{-1}(\beta)\right]^{n} \mathbf{q}\left(v_{*}, \omega_{*}\right),
\end{aligned}
$$

for any $n \in \mathbb{N}$. Further, since $\left(\psi^{-1}\left(\alpha_{1}\right)\right)^{-1} \psi^{-1}(\beta)<$ e, we get

$$
\left\|\left[\left(\psi^{-1}\left(\alpha_{1}\right)\right)^{-1} \psi^{-1}(\beta)\right]^{n}\right\| \leq\left\|\left(\psi^{-1}\left(\alpha_{1}\right)\right)^{-1} \psi^{-1}(\beta)\right\|^{n} \rightarrow \theta,
$$

as $n \rightarrow \infty$, which means that for any $c \gg \theta$ we can have $n_{0} \in \mathbb{N}$ such that

$$
\mathrm{q}\left(v_{*}, \omega_{*}\right) \leq\left[\left(\psi^{-1}\left(\alpha_{1}\right)\right)^{-1} \psi^{-1}(\beta)\right]^{n} \mathrm{q}\left(v_{*}, \omega_{*}\right) \ll c
$$

Thereby, by Lemma 1.6 it follows that $\mathbf{q}\left(v_{*}, \omega_{*}\right)=\theta$, and $v_{*}$ is the unique fixed point of the mappings $\mathcal{U}$ and $\mathcal{V}$.

Corollary 2.6 Let $\left(\mathrm{X}, \mathrm{q}, \mathcal{E}_{d}\right)$ be a complete $\Delta$-symmetric $q C M S$ over $\mathcal{E}_{d}$ and $\mathrm{P}_{\mathcal{E}_{d}}^{1}$. Suppose that $\psi: \mathrm{P}_{\mathcal{E}_{d}}^{1} \rightarrow \mathrm{P}_{\mathcal{E}_{d}}^{1}$ is a $\psi$-operator and $\mathcal{U}: \mathrm{X} \rightarrow \mathrm{X}$ is a mapping satisfying the condition

$$
\alpha_{1} \psi\left(\mathbf{q}(\mathcal{U} v, \mathcal{U} \omega)+\alpha_{2} \psi(\mathbf{q}(v, \mathcal{U} v))+\alpha_{3} \psi(\mathbf{q}(\omega, \mathcal{U} \omega)) \leq \beta \psi(\mathbf{q}(v, \omega))\right.
$$

for all $v, \omega \in \mathrm{X}$ with $\mathrm{q}(v, \omega)>\theta$, where

$$
\theta<\psi^{-1}\left(\alpha_{2}\right), \theta \leq \psi^{-1}(\beta)<\psi^{-1}\left(\alpha_{1}\right)+\psi^{-1}\left(\alpha_{2}\right)+\psi^{-1}\left(\alpha_{3}\right),
$$

in $\mathrm{P}_{\mathcal{E}_{d}}^{1}$. Then $\mathcal{U}$ has a fixed point. Moreover, if $\psi^{-1}(\beta)<\psi^{-1}\left(\alpha_{1}\right)$ then the fixed point is unique.

Proof Put $\mathcal{U}=\mathcal{V}$ in Theorem 2.5. 
Theorem 2.7 Let $\left(\mathrm{X}, \mathrm{q}, \mathcal{E}_{d}\right)$ be a complete $\Delta$-symmetric $q C M S$ over $\mathcal{E}_{d}$ and $\mathrm{P}_{\mathcal{E}_{d}}^{1}$ be a normal algebra cone in $\mathcal{E}_{d}$. Suppose that $\psi: \mathrm{P}_{\mathcal{E}_{d}}^{1} \rightarrow \mathrm{P}_{\mathcal{E}_{d}}^{1}$ is a $\psi$-operator and $\mathcal{U}, \mathcal{V}: \mathrm{X} \rightarrow \mathrm{X}$ are mappings satisfying the conditions

$$
\begin{aligned}
& \alpha_{1} \psi\left(\mathrm{q}(\mathcal{U} v, \mathcal{V} \omega)+\alpha_{2} \psi(\mathrm{q}(\omega, \mathcal{U} v))+\alpha_{3} \psi(\mathrm{q}(v, \mathcal{V} \omega)) \leq \beta \psi(\mathrm{q}(v, \omega))\right. \\
& \alpha_{1} \psi\left(\mathrm{q}(\mathcal{V} v, \mathcal{U} \omega)+\alpha_{2} \psi(\mathrm{q}(\omega, \mathcal{V} v))+\alpha_{3} \psi(\mathrm{q}(v, \mathcal{U} \omega)) \leq \beta \psi(\mathrm{q}(v, \omega))\right.
\end{aligned}
$$

for all $v, \omega \in \mathrm{X}$ with $\mathrm{q}(v, \omega)>\theta$, where $\theta \leq \psi^{-1}(\beta)+(\Delta-\mathrm{e}) \psi^{-1}\left(\alpha_{3}\right) \leq \psi^{-1}\left(\alpha_{1}\right)$ in $\mathrm{P}_{\mathcal{E}_{d}}^{1}$. Then $\mathcal{U}$ and $\mathcal{V}$ have a common fixed point.

Proof Let $\left\{v_{m}\right\}$ be the sequence in X defined by (7). Letting $v=v_{2 m}$ and $\omega=v_{2 m+1}$, by (16), we have

$$
\begin{aligned}
& \alpha_{1} \psi\left(\mathrm{q}\left(\mathcal{U} \tau_{2 m}, \mathcal{V} \tau_{2 m+1}\right)\right)+\alpha_{2} \psi\left(\mathrm{q}\left(v_{2 m+1}, \mathcal{U} \tau_{2 m}\right)\right)+\alpha_{3} \psi\left(\mathrm{q}\left(v_{2 m}, \mathcal{V} \tau_{2 m+1}\right)\right) \\
& \quad \leq \beta \psi\left(\mathrm{q}\left(\tau_{2 m}, \mathcal{V}_{\tau_{2 m+1}}\right)\right) .
\end{aligned}
$$

Moreover, by applying $\psi^{-1}$, and taking into account the properties of it,

$$
\begin{aligned}
& \psi^{-1}\left(\alpha_{1}\right) \mathrm{q}\left(v_{2 m+1}, v_{2 m+2}\right)+\psi^{-1}\left(\alpha_{2}\right) \mathrm{q}\left(\tau_{2 m+1}, v_{2 m+1}\right)+\psi^{-1}\left(\alpha_{3}\right) \mathrm{q}\left(v_{2 m}, v_{2 m+2}\right) \\
& \quad \leq \psi^{-1}(\beta) \mathrm{q}\left(v_{2 m}, v_{2 m+1}\right)
\end{aligned}
$$

and using the triangle inequality we get

$$
\mathbf{q}\left(\tau_{2 m+1}, \tau_{2 m+2}\right)-\mathbf{q}\left(\tau_{2 m+1}, \tau_{2 m}\right) \leq \mathbf{q}\left(\tau_{2 m}, \tau_{2 m+2}\right)
$$

and then

$$
\begin{aligned}
& \psi^{-1}\left(\alpha_{1}\right) \mathbf{q}\left(\tau_{2 m+1}, v_{2 m+2}\right)+\psi^{-1}\left(\alpha_{3}\right)\left[\mathbf{q}\left(v_{2 m+1}, v_{2 m+2}\right)-\mathbf{q}\left(v_{2 m+1}, v_{2 m}\right)\right] \\
& \quad \leq \psi^{-1}(\beta) \mathbf{q}\left(\tau_{2 m}, v_{2 m+1}\right),
\end{aligned}
$$

which is equivalent with

$$
\left(\psi^{-1}\left(\alpha_{1}\right)+\psi^{-1}\left(\alpha_{3}\right)\right) \mathbf{q}\left(v_{2 m+1}, v_{2 m+2}\right) \leq \psi^{-1}(\beta) \mathbf{q}\left(v_{2 m}, v_{2 m+1}\right)+\psi^{-1}\left(\alpha_{3}\right) \mathbf{q}\left(\tau_{2 m+1}, v_{2 m}\right) .
$$

Further, since the $q C M S$ is $\Delta$-symmetric, there exists an invertible element $\Delta \in \mathcal{E}$ such that $\mathrm{q}\left(\tau_{2 m+1}, \tau_{2 m}\right) \leq \Delta \mathrm{q}\left(\tau_{2 m}, \tau_{2 m+1}\right)$, for all $m \in \mathbb{N}$ and then we have

$$
\left(\psi^{-1}\left(\alpha_{1}\right)+\psi^{-1}\left(\alpha_{3}\right)\right) q\left(\tau_{2 m+1}, \tau_{2 m+2}\right) \leq\left(\psi^{-1}(\beta)+\Delta \psi^{-1}\left(\alpha_{3}\right)\right) q\left(\tau_{2 m}, \tau_{2 m+1}\right) .
$$

Therefore,

$$
\mathbf{q}\left(\tau_{2 m+1}, v_{2 m+2}\right) \leq\left(\psi^{-1}\left(\alpha_{1}\right)+\psi^{-1}\left(\alpha_{3}\right)\right)^{-1}\left(\psi^{-1}(\beta)+\Delta \psi^{-1}\left(\alpha_{3}\right)\right) \mathbf{q}\left(v_{2 m}, v_{2 m+1}\right) .
$$

On the other hand, with $v=\tau_{2 m-1}$ and $\omega=\tau_{2 m}$, the inequality (17) becomes

$$
\alpha_{1} \psi\left(\mathbf{q}\left(\mathcal{V}_{\tau_{2 m-1}}, \mathcal{U} \tau_{2 m}\right)\right)+\alpha_{2} \psi\left(\mathbf{q}\left(\tau_{2 m}, \mathcal{V}_{\tau_{2 m-1}}\right)\right)+\alpha_{3} \psi\left(\mathbf{q}\left(\tau_{2 m-1}, \mathcal{U} \tau_{2 m}\right)\right)
$$




$$
\leq \beta \psi\left(\mathrm{q}\left(\tau_{2 m-1}, v_{2 m}\right)\right)
$$

Applying $\psi^{-1}$ and keeping in mind its properties we get

$$
\begin{aligned}
& \psi^{-1}\left(\alpha_{1}\right) \mathbf{q}\left(\tau_{2 m}, v_{2 m+1}\right)+\psi^{-1}\left(\alpha_{2}\right) \mathrm{q}\left(\tau_{2 m}, v_{2 m}\right)+\psi^{-1}\left(\alpha_{3}\right) \mathrm{q}\left(\tau_{2 m-1}, v_{2 m+1}\right) \\
& \quad \leq \psi^{-1}(\beta) \mathrm{q}\left(\tau_{2 m-1}, v_{2 m}\right) .
\end{aligned}
$$

Therefore, since

$$
\mathbf{q}\left(\tau_{2 m}, \tau_{2 m+1}\right)-\mathbf{q}\left(v_{2 m}, v_{2 m-1}\right) \leq \mathbf{q}\left(\tau_{2 m-1}, v_{2 m+1}\right)
$$

we have

$$
\begin{aligned}
& \psi^{-1}\left(\alpha_{1}\right) \mathrm{q}\left(\tau_{2 m}, v_{2 m+1}\right)+\psi^{-1}\left(\alpha_{3}\right) \mathrm{q}\left(\tau_{2 m}, v_{2 m+1}\right)-\psi^{-1}\left(\alpha_{3}\right) \mathrm{q}\left(\tau_{2 m}, \tau_{2 m-1}\right) \\
& \quad \leq \psi^{-1}\left(\alpha_{1}\right) \mathrm{q}\left(\tau_{2 m}, v_{2 m+1}\right)+\psi^{-1}\left(\alpha_{3}\right) \mathrm{q}\left(\tau_{2 m-1}, v_{2 m+1}\right) \\
& \leq \psi^{-1}(\beta) \mathrm{q}\left(v_{2 m-1}, v_{2 m}\right) .
\end{aligned}
$$

Thus,

$$
\left(\psi^{-1}\left(\alpha_{1}\right)+\psi^{-1}\left(\alpha_{3}\right)\right) \mathbf{q}\left(\tau_{2 m}, v_{2 m+1}\right) \leq\left(\psi^{-1}(\beta)+\Delta \psi^{-1}\left(\alpha_{3}\right)\right) q\left(v_{2 m-1}, v_{2 m}\right)
$$

and

$$
\mathrm{q}\left(\tau_{2 m}, \tau_{2 m+1}\right) \leq\left(\psi^{-1}\left(\alpha_{1}\right)+\psi^{-1}\left(\alpha_{3}\right)\right)^{-1}\left(\psi^{-1}(\beta)+\Delta \psi^{-1}\left(\alpha_{3}\right)\right) \mathrm{q}\left(v_{2 m-1}, v_{2 m}\right) .
$$

Consequently, from (18) we conclude that

$$
\mathbf{q}\left(v_{m}, v_{m+1}\right) \leq \kappa \mathbf{q}\left(v_{m-1}, v_{m}\right)
$$

for any $m \in \mathbb{N}$, where $\kappa=\left(\psi^{-1}\left(\alpha_{1}\right)+\psi^{-1}\left(\alpha_{3}\right)\right)^{-1}\left(\psi^{-1}(\beta)+\Delta \psi^{-1}\left(\alpha_{3}\right)\right)<$ e. In this case we get $\rho(\kappa)<1$ and taking into account Lemma 2.1 we can conclude that the sequence $\left\{v_{m}\right\}$ is Cauchy and moreover convergent to an element $v_{*} \in \mathrm{X}$. Therefore, for any $c \gg \theta$, there exists $m_{1} \in \mathbb{N}$ such that $\mathrm{q}\left(v_{2 m}, v_{*}\right) \ll c, \mathrm{q}\left(v_{2 m-1}, v_{*}\right) \ll c$. We claim that $v^{*}$ is a fixed point of mappings $\mathcal{V}$ and $\mathcal{U}$. Indeed, from (16) and (17) we have

$$
\begin{aligned}
& \alpha_{1} \psi\left(\mathbf{q}\left(v_{2 m+1}, \mathcal{V}_{v_{*}}\right)\right)+\alpha_{2} \psi\left(\mathrm{q}\left(v_{*}, \tau_{2 m+1}\right)\right)+\alpha_{3} \psi\left(\mathrm{q}\left(\tau_{2 m}, \mathcal{V}_{v_{*}}\right)\right) \\
& \quad=\alpha_{1} \psi\left(\mathbf{q}\left(\mathcal{U} v_{2 m}, \mathcal{V} v_{*}\right)\right)+\alpha_{2} \psi\left(\mathbf{q}\left(v_{*}, \mathcal{U} \tau_{2 m}\right)\right)+\alpha_{3} \psi\left(\mathbf{q}\left(v_{2 m}, \mathcal{V} v_{*}\right)\right) \leq \beta \psi\left(\mathrm{q}\left(\tau_{2 m}, v_{*}\right)\right)
\end{aligned}
$$

which becomes (by applying $\psi^{-1}$ )

$$
\begin{aligned}
& \left.\psi^{-1}\left(\alpha_{1}\right) \mathbf{q}\left(v_{2 m+1}, \mathcal{V} v_{*}\right)+\psi^{-1}\left(\alpha_{2}\right) \mathbf{q}\left(v_{*}, v_{2 m+1}\right)+\psi^{-1}\left(\alpha_{3}\right)\right) \mathbf{q}\left(v_{2 m}, \mathcal{V} v_{*}\right) \\
& \quad \leq \psi^{-1}(\beta) \mathbf{q}\left(v_{2 m}, v_{*}\right) .
\end{aligned}
$$


But since $\mathbf{q}\left(v_{*}, \mathcal{V}_{v_{*}}\right) \leq \mathbf{q}\left(v_{*}, v_{2 m}\right)+\mathbf{q}\left(v_{2 m}, \mathcal{V}_{v_{*}}\right)$ and also

$$
\mathbf{q}\left(v_{*}, \mathcal{V}_{v_{*}}\right) \leq \mathrm{q}\left(v_{*}, v_{2 m+1}\right)+\mathbf{q}\left(v_{2 m+1}, \mathcal{V} v_{*}\right)
$$

we get

$$
\begin{aligned}
& \left(\psi^{-1}\left(\alpha_{1}\right)+\psi^{-1}\left(\alpha_{3}\right)\right) \mathbf{q}\left(v_{*}, \mathcal{V} v_{*}\right)-\psi^{-1}\left(\alpha_{1}\right) \mathbf{q}\left(v_{*}, v_{2 m+1}\right)-\psi^{-1}\left(\alpha_{3}\right) \mathbf{q}\left(v_{*}, v_{2 m}\right) \\
& \quad \leq \psi^{-1}(\beta) \mathbf{q}\left(v_{2 m}, v_{*}\right) .
\end{aligned}
$$

Thereupon,

$$
\mathrm{q}\left(v_{*}, \mathcal{V}_{v_{*}}\right) \leq\left(\psi^{-1}\left(\alpha_{1}\right)+\psi^{-1}\left(\alpha_{3}\right)\right)^{-1}\left(\psi^{-1}(\beta)+\Delta \psi^{-1}\left(\alpha_{2}\right)+\Delta \psi^{-1}\left(\alpha_{3}\right)\right) c
$$

which (by taking into account Lemma 1.6) shows us that $\mathbf{q}\left(v_{*}, \mathcal{V} v_{*}\right)=\theta$.

Now, similarly, by (19), we have

$$
\begin{aligned}
& \alpha_{1} \psi\left(\mathrm{q}\left(v_{2 m}, \mathcal{U} v_{*}\right)\right)+\alpha_{2} \psi\left(\mathrm{q}\left(v_{*}, v_{2 m}\right)\right)+\alpha_{3} \psi\left(\mathrm{q}\left(v_{2 m-1}, \mathcal{U} v_{*}\right)\right) \\
& \quad \leq \alpha_{1} \psi\left(\mathrm{q}\left(\mathcal{V}_{\tau_{2 m-1}}, \mathcal{U} v_{*}\right)\right)+\alpha_{2} \psi\left(\mathrm{q}\left(v_{*}, \mathcal{V}_{v_{2 m-1}}\right)\right)+\alpha_{3} \psi\left(\mathrm{q}\left(v_{2 m-1}, \mathcal{U} v_{*}\right)\right) \\
& \quad \leq \beta \psi\left(\mathrm{q}\left(v_{2 m-1}, v_{*}\right)\right),
\end{aligned}
$$

which is equivalent with

$$
\psi^{-1}\left(\alpha_{1}\right) \mathbf{q}\left(v_{2 m}, \mathcal{U} v_{*}\right)+\psi^{-1}\left(\alpha_{2}\right) \mathbf{q}\left(v_{*}, v_{2 m}\right)+\psi^{-1}\left(\alpha_{3}\right) \mathbf{q}\left(v_{2 m-1}, \mathcal{U} v_{*}\right) \leq \psi^{-1}(\beta) \mathbf{q}\left(v_{2 m-1}, v_{*}\right) .
$$

Moreover, by using the triangle inequality,

$$
\begin{aligned}
& \left(\psi^{-1}\left(\alpha_{1}\right)+\psi^{-1}\left(\alpha_{3}\right)\right) \mathbf{q}\left(v_{*}, \mathcal{U} v_{*}\right)-\psi^{-1}\left(\alpha_{1}\right) \mathbf{q}\left(v_{*}, v_{2 m}\right)-\psi^{-1}\left(\alpha_{3}\right) \mathbf{q}\left(v_{*}, v_{2 m-1}\right) \\
& \quad \leq \psi^{-1}(\beta) \mathbf{q}\left(v_{2 m-1}, v_{*}\right)
\end{aligned}
$$

then

$$
\begin{aligned}
\mathrm{q}\left(v_{*}, \mathcal{U} v_{*}\right) & \leq\left(\psi^{-1}\left(\alpha_{1}\right)+\psi^{-1}\left(\alpha_{3}\right)\right)^{-1}\left(\psi^{-1}(\beta)+\Delta \psi^{-1}\left(\alpha_{1}\right)+\Delta \psi^{-1}\left(\alpha_{3}\right)\right) \mathrm{q}\left(v_{2 m-1}, v_{*}\right) \\
& \leq\left(\psi^{-1}\left(\alpha_{1}\right)+\psi^{-1}\left(\alpha_{3}\right)\right)^{-1}\left(\psi^{-1}(\beta)+\Delta \psi^{-1}\left(\alpha_{1}\right)+\Delta \psi^{-1}\left(\alpha_{3}\right)\right) c
\end{aligned}
$$

Thus, $\mathbf{q}\left(v_{*}, \mathcal{U} v_{*}\right)=\theta$ and $v_{*}$ is a common fixed point of the mappings $\mathcal{V}$ and $\mathcal{U}$.

Corollary 2.8 Let $\left(\mathrm{X}, \mathrm{q}, \mathcal{E}_{d}\right)$ be a complete $\Delta$-symmetric $q C M S$ over $\mathcal{E}_{d}$ and $\mathrm{P}_{\mathcal{E}_{d}}^{1}$ be a normal algebra cone in $\mathcal{E}_{d}$. Assume $\psi: \mathrm{P}_{\mathcal{E}_{d}}^{1} \rightarrow \mathrm{P}_{\mathcal{E}_{d}}^{1}$ is a $\psi$-operator and $\mathcal{U}: \mathrm{X} \rightarrow \mathrm{X}$ is satisfying the condition

$$
\alpha_{1} \psi\left(\mathbf{q}(\mathcal{U} v, \mathcal{U} \omega)+\alpha_{2} \psi(\mathrm{q}(\omega, \mathcal{U} v))+\alpha_{3} \psi(\mathrm{q}(v, \mathcal{U} \omega)) \leq \beta \psi(\mathrm{q}(v, \omega))\right.
$$

for all $v, \omega \in \mathrm{X}$ with $\mathrm{q}(v, \omega)>\theta$, where $\theta \leq \psi^{-1}(\beta)+(\Delta-\mathrm{e}) \psi^{-1}\left(\alpha_{3}\right) \leq \psi^{-1}\left(\alpha_{1}\right)$ in $\mathrm{P}_{\mathcal{E}_{d}}^{1}$. Then $\mathcal{U}$ has a fixed point. 
Acknowledgements

The authors thank their universities.

\section{Funding}

We declare that funding is not applicable for our paper

\section{Availability of data and materials}

The data and material used to support the findings of this study are included within the article.

\section{Competing interests}

The authors declare that they have no competing interests.

\section{Authors' contributions}

All authors contributed equally and significantly in writing this article. All authors read and approved the final manuscript.

\section{Author details}

'Department of Mathematics and Computer Sciences, Transilvania University of Brasov, Brasov, Romania. ${ }^{2}$ Department of Mathematics, Basic Science Faculty, University of Bonab, Bonab, Iran. ${ }^{3}$ Department of Mathematics, Farhangian University, Qazvin, Iran.

\section{Publisher's Note}

Springer Nature remains neutral with regard to jurisdictional claims in published maps and institutional affiliations.

Received: 26 April 2021 Accepted: 14 June 2021 Published online: 24 June 2021

\section{References}

1. Huang, L.-G., Zhang, X.: Cone metric spaces and fixed point theorems of contractive mappings. J. Math. Anal. Appl. 332(2), 1468-1476 (2007)

2. Cho, S.-H.: Fixed point theorems in complete cone metric spaces over Banach algebras. J. Funct. Spaces 2018, Article ID 9395057 (2018)

3. Huang, H.-P., Radenović, S.: Some fixed point results of generalized Lipschitz mappings on cone $b$-metric spaces over Banach algebras. J. Comput. Anal. Appl. 20, 566-583 (2016)

4. Janković, S., Kadelburg, Z., Radenović, S.: On cone metric spaces: a survey. Nonlinear Anal., Theory Methods Appl. 4(7), 2591-2601 (2011)

5. Abdeljawad, T., Turkoglu, D., Abuloha, M.: Some theorems and examples of cone Banach spaces. J. Comput. Anal. Appl. 12(4), 739-753 (2010)

6. Afshari, H., Alsulami, H.H., Karapinar, E.: On the extended multivalued Geraghty type contractions. J. Nonlinear Sci. Appl. 9, 4695-4706 (2016). https://doi.org/10.22436/jnsa.009.06.108

7. Afshari, H., Rezapour, Sh., Shahzad, N.: Absolute retractivity of the common fixed points set of two multifunctions. Topol. Methods Nonlinear Anal. 40, 429-436 (2012)

8. Karapınar, E., Tukoglu, A.D.: Best approximations theorem for a couple in cone Banach space. Fixed Point Theory Appl. 2010, Article ID 784578 (2010)

9. Abdeljawad, T., Karapınar, E., Taş, K.: Common fixed point theorems in cone Banach spaces. Hacet. J. Math. Stat. 40(2), 211-217 (2011)

10. Abdeljawad, T., Karapınar, E.: A common fixed point theorem of a Gregus type on convex cone metric spaces. J. Comput. Anal. Appl. 13(4), 609-621 (2011)

11. Karapınar, E.: Some nonunique fixed point theorems of Ciric type on cone metric spaces. Abstr. Appl. Anal. 2010, Article ID 123094 (2010). https://doi.org/10.1155/2010/123094

12. Karapınar, K.: Couple fixed point theorems for nonlinear contractions in cone metric spaces. Comput. Math. Appl. 59(12), 3656-3668 (2010). https://doi.org/10.1016/j.camwa.2010.03.062

13. Karapınar, E.: Fixed point theorems in cone Banach spaces. Fixed Point Theory Appl. 2009, Article ID 609281 (2009). https://doi.org/10.1155/2009/609281

14. Abdeljawad, T., Karapınar, E.: Quasi-cone metric spaces and generalizations of Caristi Kirk's theorem. Fixed Point Theory Appl. 2009, Article ID 574387 (2009). https://doi.org/10.1155/2009/574387

15. Karapınar, E., Fulga, A.: On Wong type contractions. Mathematics 8(4), 649 (2020). https://doi.org/10.3390/math8040649

16. Marasi, H.R., Afshari, H., Daneshbastam, M., Zhai, C.B.: Fixed points of mixed monotone operators for existence and uniqueness of nonlinear fractional differential equations. J. Contemp. Math. Anal. 52, 8C13 (2017)

17. Liu, H., Xu, S.: Cone metric spaces with Banach algebras and fixed point theorems of generalized Lipschitz mappings. Fixed Point Theory Appl. 2013, 320 (2013)

18. LV, X.Y., Feng, Y.Q.: Some fixed point theorems for Reich type contraction in generalized metric spaces. J. Math. Anal. 9(5), 80-88 (2018)

19. Shah, M.H., Simić, S., Hussain, N., Sretenović, A., Radenović, S.: Common fixed points for occasionally weakly compatible pairs on cone metric type spaces. J. Comput. Anal. Appl. 14(2), 290-297 (2012)

20. Shojaat, H., Afshari, H., Asgari, M.S.: A new class of mixed monotone operators with concavity and applications to fractional differential equations. TWMS J. Appl. Eng. Math. 11(1), 122-133 (2021)

21. Yosida, K: Functional Analysis. Beijing World Publishing Corporation (1999)

22. Rudin, W.: Functional Analysis and Its Applications. McGraw-Hill, New York (1991)

23. Ahmed, A., Salunke, J.N.: Algebra cone generalized $b$-metric space over Banach algebra and fixed point theorems of generalized Lipschitz mappings. Asian-Eur. J. Math. 11(3), 11 (2018)

24. Hussain, N., Shah, M.H.: KKM mappings in cone b-metric spaces. Comput. Math. Appl. 62(4), 1677-1684 (2011)

25. Shaddad, F., Noorani, M., Salmi, Md.: Fixed point results in quasi-cone metric spaces. Abstr. Appl. Anal. 2013, Article ID $303626(2013)$ 